\title{
Interaction of $\alpha_{2}$-Macroglobulin with Trypsin, Chymotrypsin, Plasmin, and Papain ${ }^{1}$
}

\author{
JAMES B. HOWELL, THOMAS BECK, BRUCE BATES, \\ AND MARGARET J. HUNTER ${ }^{2}$
}

\author{
Department of Biological Chemistry, The University of Michigan Medical School and \\ The Biophysics Research Division, Institute of Science and Technology, \\ The University of Michigan, Ann Arbor, Michigan 48109
}

Received March 23, 1982, and in revised form July 7, 1982

The interaction $\alpha_{2}$-macroglobulin with four proteinases has been investigated by binding assays and by gel electrophoresis. At $\mathrm{pH} 7.65$ the binding ratios of the proteinase- $\alpha_{2}$-macroglobulin complexes were found to be 2:1 (trypsin and papain), 1.4:1 (chymotrypsin), and 1:1 (plasmin). The progressive decrease in the stoichiometry of the three seryl proteinase complexes was paralleled by a concomitant decrease in the proteinase-dependent specific cleavage of the $\alpha_{2}$-macroglobulin peptide chains. Rate studies have shown that the relative rates of reaction of the proteinases with $\alpha_{2}{ }^{-}$ macroglobulin also varied greatly: papain $>$ trypsin $>$ chymotrypsin $>$ plasmin. The data suggest that the ability of a proteinase to saturate the second proteinase binding site is a reflection of its ability to bind to $\alpha_{2}$-macroglobulin and cleave the second pair of scissile $\alpha_{2}$-macroglobulin peptide bonds before the $\alpha_{2}$-macroglobulin has undergone the conformational change initiated by the formation of the 1:1 proteinase $\alpha_{2}$-macroglobulin complex.

Human plasma $\alpha_{2}$-macroglobulin is a plasma glycoprotein of approximately 720,000 daltons. The molecule is a tetramer composed of identical subunits linked in pairs by disulfide bonds. Two disulfide pairs associate noncovalently to form the native tetramer $(1,2)$.

Although $\alpha_{2}$-macroglobulin is generally classified as a proteinase inhibitor, certain of its biochemical characteristics are strikingly different from those of other members of this group. Two such deviant

\footnotetext{
${ }^{1}$ This work was supported in part by National Institutes of Health Research Grant HL-09739-12 and by a Research Grant from the Horace H. Rackham School of Graduate Studies, The University of Michigan.

${ }^{2}$ Author to whom all correspondence should be sent: Biophysics Research Division, Room 1256, Institute of Science and Technology, The University of Michigan, 2200 Bonisteel Blvd., Ann Arbor, Mich. 48109.
}

properties are the ability of $\alpha_{2}$-macroglobulin to bind an unusually wide variety of endopeptidases (seryl, cysteinyl, aspartyl, and metal proteinases) $(3,4)$ and the continued hydrolytic activity of $\alpha_{2}$-macroglobulin-bound proteinases to small molecule, but not macromolecule, substrates (5). The binding of proteinase by $\alpha_{2}$-macroglobulin is accompanied by a specific cleavage of the $\alpha_{2}$-macroglobulin subunit chains $(1,2,4)$ although in the absence of denaturants and small molecule thiols the $\alpha_{2}$-macroglobulin-proteinase complex remains intact.

Literature values for proteinase: $\alpha_{2}$-macroglobulin binding ratios fall between 1 and 2 mol proteinase bound per mole $\alpha_{2}$-macroglobulin $(3,4,6-9)$. The reported values show an unusual degree of variability. If we assume that $\alpha_{2}$-macroglobulin intrinsically contains two binding sites for all proteinases (a fair assumption in view of 
the dimer characteristics of the molecule), the low binding ratios which have been reported for certain proteinases are presumably a reflection of ancillary events which restrict the ability of the $\alpha_{2}$-macroglobulin to bind the second proteinase molecule.

The studies on the interaction of $\alpha_{2}-$ macroglobulin with trypsin, chymotrypsin, plasmin, and papain which are reported in this paper were designed to investigate the factors responsible for the variability in the binding ratios of these four $\alpha_{2}$-macroglobulin-proteinase complexes.

\section{MATERIALS AND METHODS}

TPCK trypsin and $3 \times$ crystallized, salt-free $\alpha$-chymotrypsin were obtained from Worthington Biochemical Corporation. Glu-plasminogen was prepared from fresh human plasma by affinity chromatography on a lysine-Sepharose $4 B$ column (10) in the presence of aprotinin. Aprotinin-free plasminogen was converted to plasmin by incubation for $1 \mathrm{~h}$ at $25^{\circ} \mathrm{C}$ with urokinase-Sepharose $4 \mathrm{~B}$ (11). The resultant plasmin preparation showed two bands on SDS $^{8}$-polyacrylamide gel electrophoresis; a major plasmin band and a minor band which had the same mobility as Glu-plasminogen. Papain which had been purified by the method of Burke et al. (12) was a gift from Dr. Jules Shafer, Department of Biological Chemistry, University of Michigan Medical School.

Preparation of $\alpha_{\boldsymbol{r}}$ macroglobulin. Polyethylene glycol 6000 (J. T. Baker Chemical Company) was added to fresh plasma to give a concentration of $4.5 \%$. The suspension was stirred for $\mathbf{3 0} \mathrm{min}$, centrifuged for $\mathbf{3 0}$ min at $5000 \mathrm{~g}$ and the supernatant removed. Sufficient polyethylene glycol was then added to the supernatant to give a concentration of $10 \%$. The resulting suspension was stirred for $30 \mathrm{~min}$, and centrifuged as before. The precipitate was washed with 0.5 plasma volume of $10 \%$ polyethylene glycol in $0.15 \mathrm{M} \mathrm{NaCl}$, recentrifuged, and the washed precipitate was dissolved in $30 \mathrm{ml}$ of $0.15 \mathrm{M} \mathrm{NaCl}$. These procedures were conducted in plastic containers $\left(4^{\circ} \mathrm{C}\right)$.

The washed 4.5-10\% precipitate was applied to a $5 \times 85-\mathrm{cm}$ gel filtration column containing LKB Ultrogel AcA 22. The column was eluted with $0.15 \mathrm{M}$ $\mathrm{NaCl}, 0.015$ M Tris buffer solution which had been adjusted to $\mathrm{pH} 7.0$ with sodium carbonate. The $\alpha_{2}-$ macroglobulin eluate fraction (as determined by

\footnotetext{
s Abbreviations used: SDS, sodium dodecyl sulfate; L-BA PNA, $\boldsymbol{N}$ - $\alpha$-benzoyl-L-arginine- $\boldsymbol{p}$-nitroanilide; BTEE, $N$-benzoyl-L-tyrosine ethyl ester.
}

trypsin-binding activity) was pooled, concentrated, and applied to a hemoglobin-Sepharose $4 B$ affinity column which had been prepared by the general method of Klein and Mihaesco (13). The resultant haptoglobin-free $\alpha_{2}$-macroglobulin fraction was pooled and concentrated, and contaminating immunoglobulins were removed from the $\alpha_{2}$-macroglobulin fraction by preparative electrophoresis at $\mathrm{pH} 8.2$ in a Brinkman Instrument electrophoretic separator, Brinkman Model FF. The $\alpha_{2}$-macroglobulin fraction was pooled, concentrated, and reapplied to the UItrogel AcA 22 column to obtain the purified $\alpha_{2}$-macroglobulin preparation.

As determined by gel double diffusion against antisera to whole plasma, $\alpha_{2}$-antitrypsin, antithrombin III, inter- $\alpha$-trypsin inhibitor, immunoglobulin $\lambda$ and * chains, antichymotrypsin, haptoglobin, and albumin (Behring Diagnostics), the $\alpha_{2}$-macroglobulin preparation was essentially free of other plasma proteins. SDS-gel electrophoresis of overloaded $4 \%$ polyacrylamide gels showed the presence of one major high-molecular-weight band with very faint traces of a lower-molecular-weight contaminant.

The molecular weight of $\alpha_{2}$-macroglobulin was assumed to be 720,000 (14).

The $\alpha_{8}$ macroglobulin proteinase interaction. The activities of the trypsin and plasmin preparations were determined by the active site titration method of Chase and Shaw $(15,16)$ using $p$-nitrophenyl- $p$ guanidinobenzoate as substrate. The activity of the chymotrypsin preparations was determined by the active site titration method of Bender et al. (17) using p-nitrophenyl acetate as substrate. The activity of the papain preparation was determined in two assay systems; one procedure used $N$ - $\alpha$-benzoyl-L-arginine$p$-nitroanilide (L-BAPNA) as substrate (18), the other 2,Z-dipyridyl disulfide (Aldrich Chemical $C o$.) as an active site titrant (19).

All proteinase concentrations given in this paper refer to "active" concentrations.

The stoichiometry of binding of trypsin to $\alpha_{2}$-macroglobulin was determined by adding increasing amounts of $\alpha_{2}$-macroglobulin to a constant amount of trypsin ( $\alpha_{2}$-macroglobulin:trypsin molar ratios of 0 to 1.07), adding soybean trypsin inhibitor to inactivate any unbound trypsin and then measuring the ability of the $\alpha_{2}$-macroglobulin-bound trypsin to catalyze the hydrolysis of the low-molecular-weight substrate BAPNA (20).

The stoichiometry of the plasmin- $\alpha_{2}$-macroglobulin complex was determined using an assay procedure identical to that employed in the assay of trypsin: $\alpha_{2}-$ macroglobulin reaction mixtures except that BAPNA was replaced by D-Val-Leu-Lys-NH- $\phi-\mathrm{NO}_{2}, \mathrm{~S}-2251$ (A. B. Kabi, Greenwich, Conn.). BAPNA is a relatively poor substrate for plasmin.

The stoichiometry of the chymotrypsin- $\alpha_{2}$-macroglobulin complex was determined by a method sim- 
ilar to that employed in the trypsin-binding measurements. In this procedure, BAPNA was replaced by the chymotrypsin substrate $N$-benzoyl-L-tyrosine ethyl ester (BTEE) and soybean trypsin inhibitor was replaced by $\alpha_{1}$-antitrypsin since chymotrypsin is not inactivated by soybean trypsin inhibitor but forms an inactive 1:1 complex with $\alpha_{1}$-antitrypsin. The antitrypsin was prepared by the method of Bloom and Hunter (21).

The assay procedures used to measure the binding ratio of the $\alpha_{2}$-macroglobulin-papain complex were somewhat different from those employed in the stoichiometry measurements on the other three proteinases since no macromolecular papain inhibitor was available. In one procedure, $\alpha_{2}$-macroglobulin:papain reaction mixtures (papain: $\alpha_{2}$-macroglobulin molar ratios of 0.8 to 15) were incubated with Azocoll (Calbiochem), an insoluble powdered cowhide to which a red dye had been covalently linked. Soluble Azocoll hydrolysis products were released in the presence of free but not $\alpha_{2}$-macroglobulin-bound papain. The $\alpha_{2}$ macroglobulin:papain stoichiometry was also determined by monitoring the ability of various $\alpha_{2}$-macroglobulin:papain reaction mixtures to bind trypsin using BAPNA as substrate. This procedure depended on (i) the relatively low activity of papain toward BAPNA, the rate of the papain-catalyzed hydrolysis being less than one-fifth that of the trypsin-catalyzed hydrolysis, and (ii) the observation that saturating $\alpha_{2}$-macroglobulin with papain resulted in a complex which was unable to bind trypsin.

SDS-gel electrophoresis under reducing and nonreducing conditions was performed by the method of Weber and Osborn (22).

\section{RESULTS}

The $\alpha_{2}$-macroglobulin-trypsin complex. The binding of trypsin by $\alpha_{2}$-macroglobulin was determined in the presence and absence of $7.5 \mathrm{~mm}$ EDTA or $3 \mathrm{~mm}$ EGTA at $\mathrm{pH} 5(0.05 \mathrm{M}$ acetate buffer $), \mathrm{pH} 7.0(0.05$ M phosphate buffer), $\mathrm{pH} 7.65$ (0.05 M Tris), and $\mathrm{pH} 9.0(0.05 \mathrm{M}$ borate buffer $)$ at $25^{\circ} \mathrm{C}$. After a $60-\mathrm{min}$ incubation period the $\mathrm{pH}$ of 3:1 trypsin: $\alpha_{2}$-macroglobulin reaction mixtures was adjusted to 7.65 by the addition of $0.1 \mathrm{M}$ Tris buffer. Control studies showed that $\alpha_{2}$-macroglobulin was not inactivated by this long incubation period at pH 5-9. Excess soybean trypsin inhibitor was added to the reaction mixtures which were then assayed for $\alpha_{2}$-macroglobulinbound trypsin by the BAPNA assay procedure. In all cases the stoichiometry of the complex was found to be 1.9-2.0:1 (Fig. 1).

The stoichiometry of binding was also investigated by SDS-polyacrylamide gel electrophoresis in the presence and absence of 2-mercaptoethanol (Fig. 2). In the presence of SDS alone no proteolysis of $\alpha_{2}$ macroglobulin to lower-molecular-weight species could be detected, even in reaction mixtures which contained excess trypsin. In the presence of SDS and mercaptoethanol, however, the $\sim 180,000$-dalton $\alpha_{2}$ macroglobulin subunits were converted to $\sim 90,000$-dalton species and the amount of the $\sim 90,000$-dalton species increased with the trypsin concentration until the trypsin: $\alpha_{2}$-macroglobulin ratio was approximately 2 at which point all the $\sim 180,000$ dalton subunits had been converted to the $\sim 90,000$-dalton species (Fig. 2). Complete hydrolysis of the four scissile $\alpha_{2}$-macroglobulin peptide bonds thus accompanied binding of two molecules of trypsin by the $\alpha_{2}$-macroglobulin.

The presence of $\mathrm{Ca}^{2+}\left(0-0.2 \mathrm{M} \mathrm{CaCl}_{2}\right)$ did not affect the stoichiometry of the $\alpha_{2}$-macroglobulin-trypsin complex. It did however decrease the rate of hydrolysis of

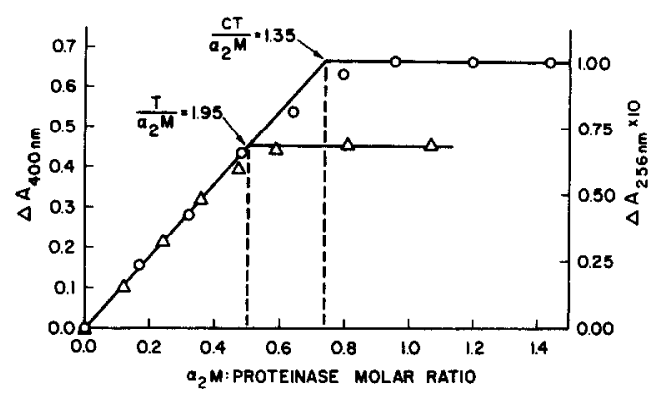

FIG. 1. Stoichiometry of $\alpha_{2}$-maeroglobulin-tryp$\sin \left(\alpha_{2} \mathrm{M}-\mathrm{T}\right)$ and $\alpha_{2}$-macroglobulin-chymotrypsin $\left(\alpha_{2} \mathrm{M}-\mathrm{CT}\right.$ ) complexes. $(\Delta) \alpha_{2} \mathrm{M}$-Bound tryptic activity $\left(A_{400 \mathrm{~nm}}\right)$ of $\alpha_{\mathrm{g}} \mathrm{M}: \mathrm{T}$ reaction mixtures in $0.025 \mathrm{M}$ Tris, pH 7.65. Trypsin concentration $=0.5 \mu \mathrm{M} ; \alpha_{2} \mathrm{M}: \mathrm{T}$ molar ratios $=0$ to 1.07 . Tryptic activity was determined by the BAPNA assay procedure in the presence of excess soybean trypsin inhibitor (STI). (O) $\alpha_{2}$ M-Bound chy motryptic activity $\left(A_{256 \mathrm{~mm}}\right)$ of $\alpha_{2} \mathrm{M}$ :CT reaction mixtures in $0.025 \mathrm{M}$ Tris, $\mathrm{pH}$ 7.65. Chymotrypsin concentration $=0.08 \mu \mathrm{M} ; \alpha_{2} \mathrm{M}: \mathrm{CT}$ molar ratios $=0$ to 1.45 . Chymotryptic activity was determined by the BTEE assay procedure in the presence of excess $\alpha_{1}$-antitrypsin. 


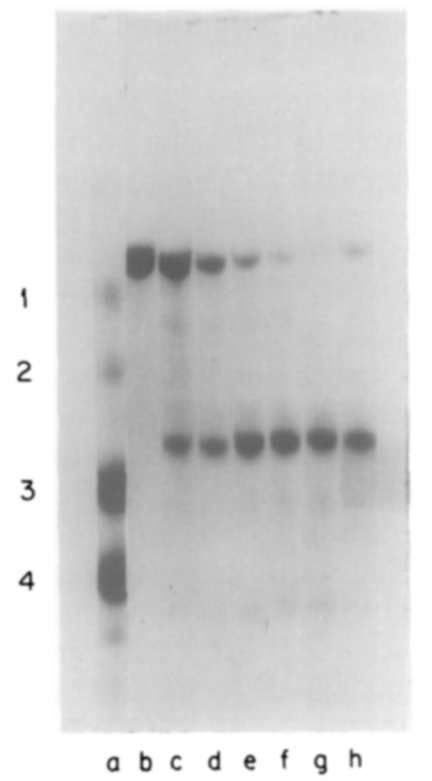

FIG. 2. Gel electrophoresis of $\alpha_{2} \mathrm{M}$ :T reaction mixtures in the presence of SDS and 2-mercaptoethanol. $10 \mu \mathrm{g} \alpha_{2} \mathrm{M}$ was incubated with various amounts of trypsin at $\mathrm{pH} 7.65,24^{\circ} \mathrm{C}$ for $10 \mathrm{~min}$ (except gel h) and the mixture incubated with $1 \% \mathrm{SDS}, 1 \%$ 2-mercaptoethanol for $60 \mathrm{~min}$ at $24^{\circ} \mathrm{C}, \mathrm{pH} 7.0$. The reaction mixtures were then diluted with 9 vol of $12 \%$ glycerol, $0.01 \%$ bromphenol blue. The reaction mixtures $\left(100 \mu \mathrm{l}, 10 \mu \mathrm{g} \alpha_{2} \mathrm{M}\right.$ ) were applied to preelectrophoresed $4 \%$ gels. The electrophoresis was stopped every 90 min to mix the top and bottom electrode solutions. The mixed solutions were then returned to the electrophoresis vessels and the electrophoresis continued. This operation was necessary due to the increase in the $\mathrm{pH}$ of the upper electrode vessel as electrophoresis continued and the extreme lability of certain $\alpha_{2} \mathrm{M}$ peptide bonds at these higher $\mathrm{pH}$ values. (a) 1 , Transferrin dimer; 2, BSA dimer; 3, transferrin monomer; 4, BSA monomer. (b) $\alpha_{2} \mathrm{M}$; (c-g) $\alpha_{2} \mathrm{M}+0.5$, $1.0,1.5,2$, and 3 eq of $T$ per mole of $\alpha_{2} M$; (h) as in gel $f$ but 24 -h incubation with $T$. The faint fastestmigrating bands in gels $c-h$ represent inactive or excess active trypsin.

BAPNA as catalyzed by $\alpha_{2}$-macroglobulinbound trypsin, a situation in sharp contrast to the $\mathrm{Ca}^{2+}$-activated hydrolysis of BAPNA by free trypsin.

Studies on the $\alpha_{2}$-macroglobulin:trypsin interaction were extended to include the serial addition of nonsaturating amounts of trypsin to $\alpha_{2}$-macroglobulin at $\mathrm{pH} 7.5$; under such conditions the binding ratio did appear to decrease somewhat. For exam- ple, when half-saturating amounts of trypsin were incubated with $\alpha_{2}$-macroglobulin for from $1 \mathrm{~min}$ to $20 \mathrm{~h}$ at $25^{\circ} \mathrm{C}$ and excess trypsin then added, the average stoichiometry of the complex was $1.5: 1$ instead of the 2:1 obtained for the complex which had been prepared by one addition of excess trypsin. If one assumes that the rate of reaction of both trypsin molecules with native $\alpha_{2}$-macroglobulin is the same (a reasonable assumption in view of the dimer characteristics of the molecule), the addition of an equivalent amount of tryp$\sin$ to $\alpha_{2}$-macroglobulin should result in a 25:50:25 mixture of $2: 1,1: 1$, and 0:1 trypsin- $\alpha_{2}$-macroglobulin complexes. If excess trypsin is added later to this reaction mixture and if the 1:1 complex cannot bind trypsin, the resultant mixture should contain a 50:50 mixture of the 2:1 and 1:1 tryp$\sin -\alpha_{2}$-macroglobulin complexes; that is, the overall binding ratio should be 1.5:1.

The $\alpha_{2}$-macroglobulin-chymotrypsin complex. In our initial studies the binding ratio of the $\alpha_{2}$-macroglobulin-chymotryp-

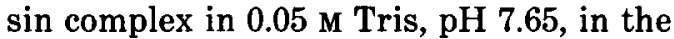
absence of $\mathrm{Ca}^{2+}$, was found to be 1.4:1 (Fig. 1) and SDS-2-mercaptoethanol gel electrophoresis of such mixtures showed that only about two-thirds of the $\sim 180,000$-dalton subunits were cleaved to $\sim 90,000$-dalton subunits even in the presence of excess chymotrypsin (Fig. 3). To inhibit the generalized proteolysis of the $\alpha_{2}$-macroglobulin which occurred during the $1-\mathrm{h}, 25^{\circ} \mathrm{C}$ incubation of the chymotrypsin: $\alpha_{2}$-macroglobulin reaction mixtures with SDS and mercaptoethanol in the absence of a small molecule inhibitor, toluene sulfonyl fluoride was added to the reaction mixtures prior to the addition of SDS and mercaptoethanol. Higher incubation temperatures (and hence shorter incubation times) could not be employed as they result in the cleavage of a specific, temperature-sensitive $\alpha_{2}$-macroglobulin peptide bond (23). Furthermore when various nonsaturating amounts of chymotrypsin were incubated with $\alpha_{2}$-macroglobulin at $\mathrm{pH} 7.65$ for 10 min and excess chymotrypsin was then added, the stoichiometries of the complexes were significantly lower (minimum chymotrypsin: $\alpha_{2}$-macroglobulin 


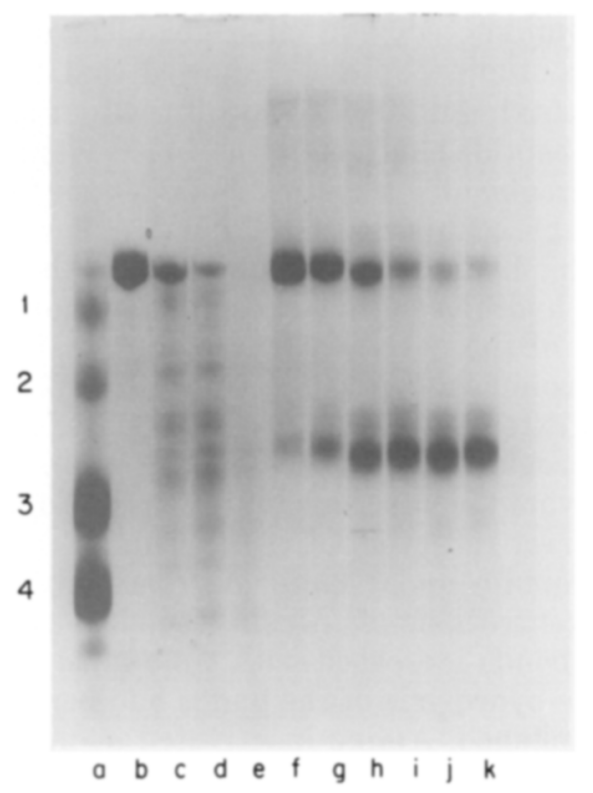

Fig. 3. Gel electrophoresis of $\alpha_{2} \mathrm{M}$ :CT reaction mixtures in the presence of SDS and 2-mercaptoethanol. The samples were treated as described in Fig. 2. In gel $\mathrm{b}$ and gels $\mathrm{f}-\mathrm{k}$ the $\alpha_{2} \mathrm{M}$ :CT mixtures were incubated with $0.002 \mathrm{M}$ toluene sulfonyl fluoride (TSF) for 30 min just prior to the addition of the SDS and 2mercaptoethanol. (a) 1, Transferrin dimer; 2, BSA dimer, 3, transferrin monomer; 4 , BSA monomer. (b) $\alpha_{2} \mathrm{M}+\mathrm{TSF} ;$ (c-e) $\alpha_{2} \mathrm{M}+0.5,1.0$, and 1.5 eq of CT per mole $\alpha_{2} \mathrm{M} ;(\mathrm{f}-\mathrm{j}) \alpha_{2} \mathrm{M}+0.35,0.7,1.05$, and 1.4 , and 4.2 eq of CT per mole $\alpha_{2} \mathrm{M}+$ TSF; (k) as in gel $\mathrm{i}$ except that $\alpha_{2} \mathrm{M}$ was incubated with $\mathrm{CT}$ for $24 \mathrm{~h}$ prior to the addition of the TSF. All other $\alpha_{2} \mathrm{M}: \mathrm{CT}$ incubations were for $10 \mathrm{~min}$.

binding ratio $=1.2: 1$ ) than that of a comparable reaction mixture prepared by one addition of excess chymotrypsin (chymotrypsin: $\alpha_{2}$-macroglobulin $=1.4: 1$ ) It was also observed that the saturated complex (binding ratio 1.4:1) did not bind trypsin even though the complex should not have been saturated with respect to trypsin binding sites.

The studies on the binding of chymotrypsin by $\alpha_{2}$-macroglobulin were extended to include pH, EGTA ( $3 \mathrm{~mm})$, EDTA $(7.5 \mathrm{mM})$, and $\mathrm{Ca}^{2+}(20 \mathrm{mM})$, as variables. At $\mathrm{pH} \mathrm{9.0,} \mathrm{the} \mathrm{stoichiometry} \mathrm{of} \mathrm{the} \mathrm{com-}$ plex was found to be 1.8:1 in the presence and absence of $\mathrm{Ca}^{2+}$. In the presence of $\mathrm{Ca}^{2+}$ the stoichiometry of the complex at $\mathrm{pH} 7.65$ was the same as that observed at
$\mathrm{pH}$ 9. In the absence of $\mathrm{Ca}^{2+}$ however, the stoichiometry decreased to 1.4:1. At lower $\mathrm{pH}$ values $(\mathrm{pH} 5)$ the stoichiometry was further depressed to a low of $\sim 1: 1$. When an excess amount of an equimolar mixture of trypsin and chymotrypsin was quickly added to $\alpha_{2}$-macroglobulin at $\mathrm{pH} 7.65$ and the reaction mixture assayed for tryptic activity in the presence of excess soybean trypsin inhibitor, $80 \%$ of the bound proteinase was found to be trypsin (Fig. 4). It therefore appears that the lower stoichiometry of the chymotrypsin- $\alpha_{2}$-macroglobulin complex is accompanied by a considerably slower rate of binding.

The $\alpha_{2}$-macroglobulin-plasmin complex. As previously reported by Pochon (24) plasmin was found to form a 1:1 complex with $\alpha_{2}$-macroglobulin (Fig. 5). When excess trypsin was added to a series of plasmin: $\alpha_{2}$-macroglobulin reaction mixtures which varied in plasmin concentration and any excess free proteinase then inactivated by the addition of soybean trypsin inhibitor, it was observed that the trypsincatalyzed hydrolysis of BAPNA decreased with increasing plasmin concentration and was essentially zero in reaction mixtures

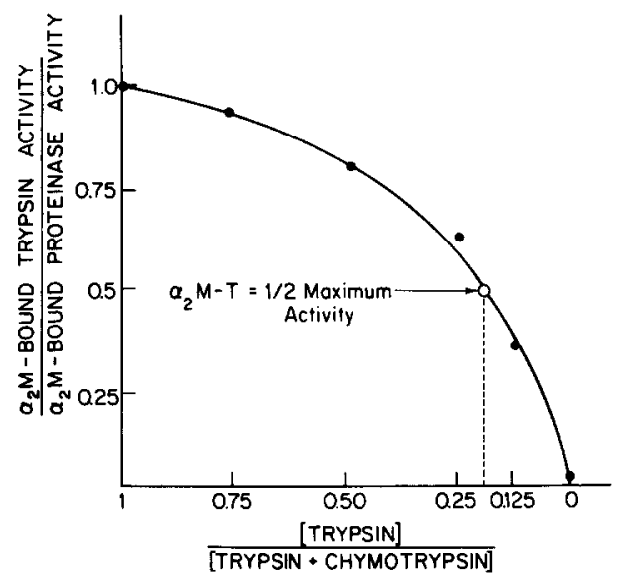

FIG. 4. The tryptic and total proteinase activities of $\alpha_{2} \mathrm{M}:(\mathrm{T}+\mathrm{CT})$ reaction mixtures. $\alpha_{2} \mathrm{M}$ concentration $=0.25 \mu \mathrm{M}$; total proteinase: $\alpha_{2} \mathrm{M}$ molar ratio $=4: 1$. The trypsin:total proteinase molar ratio varied from 0 to $1 . \alpha_{2}$ M-Bound tryptic activity was determined by the BAPNA assay procedure in the presence of excess STI, $\alpha_{2} \mathrm{M}$-bound chymotryptic activity by the BTEE assay procedure in the presence of excess $\alpha_{1}$-antitrypsin. Buffer $=0.1 \mathrm{M}$ Tris, pH 7.65. 


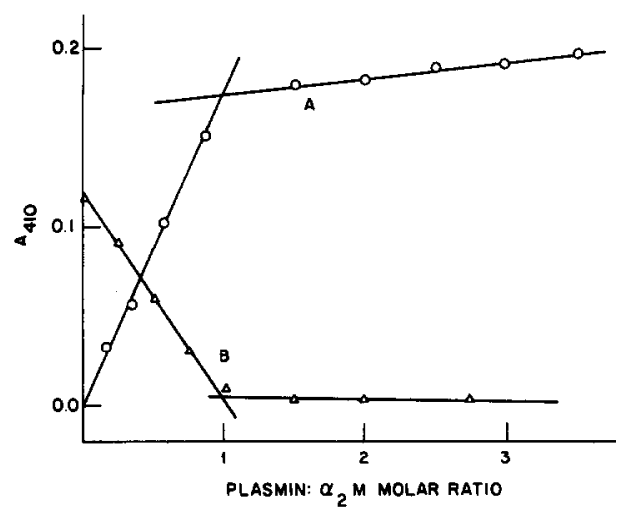

FIG. 5. The interaction of plasmin with $\alpha_{2}$ M. (A) Various amounts of $5.6 \mu \mathrm{M}$ plasmin were mixed with $1.0 \mathrm{ml}$ of $0.05 \mathrm{M}$ Tris, $\mathrm{pH} \mathrm{7.6}$, and $0.1 \mathrm{ml}$ of $1.1 \mu \mathrm{M} \alpha_{2} \mathrm{M}$. The volumes of the solutions were made up to $1.5 \mathrm{ml}$ with $0.1 \mathrm{M} \mathrm{NaCl}$. After $10 \mathrm{~min} 0.06 \mathrm{ml}$ of $1 \mathrm{mg} / \mathrm{ml}$ STI was added to the reaction mixtures to inactivate unbound plasmin. $0.5 \mathrm{ml}$ of $\mathrm{S}-2251$ (substrate) was then added to the reaction mixtures. After $10 \mathrm{~min}$ the reaction was stopped by the addition of $1 \mathrm{ml}$ of $33 \%$ acetic acid. (B) Reaction mixtures similar to those in $A$ were prepared except that $0.04 \mathrm{ml}$ of $1 \mathrm{mg} / \mathrm{ml}$ trypsin (excess) was added to the plasmin: $\alpha_{2}$-macroglobulin reaction mixtures before the STI. The substrate in these experiments was DL-BAPNA and the reactions were stopped after $15 \mathrm{~min}$ by the addition of acetic acid. The data have been corrected for the slow hydrolysis of BAPNA by $\alpha_{2} M$-bound plasmin.

that contained a plasmin: $\alpha_{2}$-macroglobulin ratio of one or greater (Fig. 5). BAPNA is such a poor substrate for plasmin relative to trypsin that the $\alpha_{2}$-macroglobulinbound plasmin in the assay solution made a negligible contribution to the observed hydrolysis of the BAPNA.

Reducing SDS-gel electrophoresis showed that a maximum of about one-half of the $\alpha_{2}$-macroglobulin chains could be cleaved by plasmin; all $\alpha_{2}$-macroglobulin chains, however, were cleaved after the subsequent addition of excess trypsin to the saturated $\alpha_{2}$-macroglobulin-plasmin complex.

This trypsin-catalyzed hydrolysis of the $\alpha_{2}$-macroglobulin peptide chains of the saturated 1:1 plasmin- $\alpha_{2}$-macroglobulin complex might appear to conflict with the binding data which showed that the saturated plasmin complex did not form a stable complex with trypsin.
Similar reducing SDS-gel electrophoresis studies, however, showed that trypsin catalyzed the cleavage of the four scissile peptide bonds of amine-inactivated $\alpha_{2}$ macroglobulin, a species that does not form a stable complex with trypsin (25). The rate of this hydrolysis was slower than that of the hydrolysis that accompanied formation of the native $\alpha_{2}$-macroglobulin-trypsin complex. Furthermore, we found that the extent of cleavage of the scissile $\alpha_{2}$ macroglobulin peptide bonds of amine-inactivated $\alpha_{2}$-macroglobulin was no longer a direct reflection of the trypsin: $\alpha_{2}$-macroglobulin molar ratio. At relatively low concentrations of trypsin, the cleavage of the bonds increased with time until complete hydrolysis of the bonds had been accomplished. Amine-inactivated $\alpha_{2}$-macroglobulin thus acted as a rather poor substrate for trypsin, rather than as an inhibitor.

Barrett $(3,26)$ has shown that the structural characteristics of amine-inactivated $\alpha_{2}$-macroglobulin differ from those of the native molecule but appear similar to those of the $\alpha_{2}$-macroglobulin-proteinase complexes. Like the amine-inactivated protein, the plasmin complex may therefore also act as a poor substrate and the trypsin-catalyzed hydrolysis of the labile $\alpha_{2}$ macroglobulin peptide bonds in the plasmin complex may be completely unrelated to the formation of a stable complex between the 1:1 plasmin- $\alpha_{2}$-macroglobulin complex and trypsin.

The rate of the $\alpha_{2}$-macroglobulin:plasmin reaction was much slower than that of the $\alpha_{2}$-macroglobulin:trypsin reaction at $\mathrm{pH}$ 7.65. When an equimolar mixture of plasmin and trypsin was quickly added to an $\alpha_{2}$-macroglobulin solution (total proteinase: $\alpha_{2}$-macroglobulin molar ratio $=4: 1$ ) and the reaction mixture assayed for trypsin and total proteinase activity after the addition of excess soybean trypsin inhibitor to inactive unbound proteinase, it was found that essentially all the $\alpha_{2}$-macroglobulin-bound proteinase was trypsin. When the plasmin:trypsin molar ratio was increased to $5: 1$ (total proteinase: $\alpha_{2}-$ macroglobulin molar ratio $=12: 1$ ) about $5 \%$ of the $\alpha_{2}$-macroglobulin-bound proteinase 
was plasmin. Total $\alpha_{2}$-macroglobulinbound proteinase activity was determined using S-2251 as substrate since this is a good substrate for both proteinases.

No $\mathrm{Ca}^{2+}$ was present in any of the $\alpha_{2-}$ macroglobulin plasmin reaction mixtures.

The $\alpha_{2}$-macroglobulin-papain complex. SMethyl-papain solutions were activated in $100 \mathrm{~mm}$ 2-mercaptoethanol, $2.5 \mathrm{~mm}$ EDTA, $\mathrm{pH} 5.8$, for $30 \mathrm{~min}\left(25^{\circ} \mathrm{C}\right)$ and passed down a Sephadex G-25 column to remove small molecule by-products. The column buffer was $0.15 \mathrm{M} \mathrm{KCl}, 2.5 \mathrm{mM}$ EDTA, $50 \mu \mathrm{M} 2$ mercaptoethanol, $\mathrm{pH} 5.8$.

Under our conditions of $\mathrm{pH}$ and buffer, the concentration of 2-mercaptoethanol must be $1 \mathrm{~mm}$ or greater to achieve full activation of $S$-methyl-papain. To retain the full activity of this activated papain at lower mercaptoethanol concentrations, the small molecule by-products of the activation reaction must be removed from the activated papain preparation. The final concentration of mercaptoethanol in papain: $\alpha_{2}$-macroglobulin reaction mixtures must be $1 \mathrm{mM}$ or lower to prevent loss of the proteinase-binding capacity of the $\alpha_{2}$-macroglobulin.

When the Azocoll assay system was used to determine the free papain concentration in $\alpha_{2}$-macroglobulin:papain reaction mixtures $(\mathrm{pH} 7.65)$ of varying papain: $\alpha_{2}-$ macroglobulin molar ratio, the stoichiometry of the saturated complex was found to be 2:1 (Fig. 6). It thus appeared that at pH 7.65 papain, like trypsin, can completely saturate both proteinase binding sites on $\alpha_{2}$-macroglobulin. These results were supported and extended using a second method to determine the $\alpha_{2}$-macroglobulin-papain stoichiometry. Papain and $\alpha_{2}$-macroglobulin were mixed in varying proportions at $\mathrm{pH} 7.65$ such that the papain: $\alpha_{2}$-macroglobulin molar ratio varied from 0 to 5 . After a $10-\mathrm{min}$ incubation period excess trypsin was added to the reaction mixtures. Any unbound trypsin was then inactivated by the addition of soybean trypsin inhibitor. At low papain concentrations the binding of trypsin was evidenced by the ability of the complex to catalyze the hydrolysis of BAPNA. As the papain: $\alpha_{2}$-macroglobulin molar ratio in-

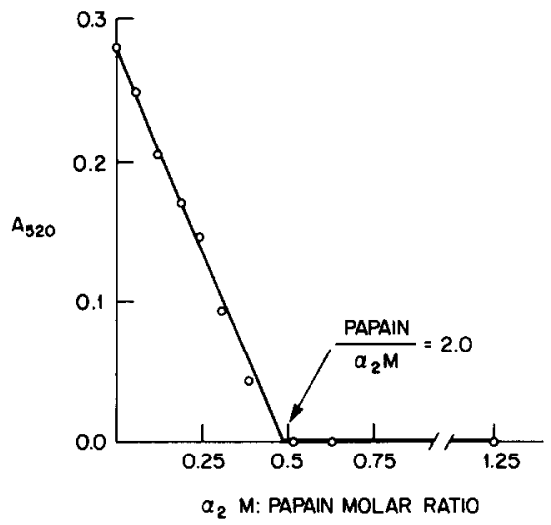

FIG. 6. The interaction of papain with $\alpha_{2} M .5 .8$ $\times 10^{-10} \mathrm{~mol}$ of papain were mixed with zero to 7.4 $\times 10^{-10} \mathrm{~mol}$ of $\alpha_{2}$-macroglobulin in $2.1 \mathrm{ml}$ of a solution containing $50 \mathrm{mM}$ Tris, $2.5 \mathrm{~mm}$ EDTA, $0.1 \mathrm{~mm}$ 2-mercaptoethanol, pH 7.65. After $10 \mathrm{~min} 2 \mathrm{ml}$ of this solution was added to $10 \mathrm{mg}$ of powdered Azocoll. The mixture was incubated for 15 min after which time the reaction was stopped by the addition of $1.0 \mathrm{ml}$ of $33 \%$ acetic acid. The $520-\mathrm{nm}$ absorbance values shown in the figure were obtained by subtacting the absorbance of a control sample (a sample to which the acetic acid had been added before addition of the Azocoll) from that of the comparable reaction mixture.

creased, the hydrolysis of BAPNA decreased to a minimum at approximately a $2: 1$ molar ratio of papain to $\alpha_{2}$-macroglobulin. At higher papain concentrations a slow increase in hydrolysis paralleled the increase in papain concentration. This slow increase resulted from the hydrolysis of BAPNA by papain since papain is not inactivated by soybean trypsin inhibitor. These data thus indicated that the saturated papain- $\alpha_{2}$-macroglobulin complex was unable to bind trypsin.

The relative rates of formation of the $\alpha_{2}$-macroglobulin-papain and $\alpha_{2}$-macroglobulin-trypsin complexes were determined by quickly mixing equimolar amounts of papain and trypsin and adding excess proteinase to the $\alpha_{2}$-macroglobulin solution at $\mathrm{pH} 7.65$ in the presence of $1 \mathrm{~mm}$ 2-mercaptoethanol. These low mercaptoethanol concentrations did not measureably affect the interaction of trypsin with $\alpha_{2}$-macroglobulin. Any unbound trypsin was then inhibited by the addition of excess soybean trypsin inhibitor. Bound and 
unbound papain were inhibited by the addition of excess iodoacetate (10 $\mathrm{mM})$. Iodoacetate did not depress the hydrolytic activity of trypsin nor did it diminish the proteinase-binding ability of $\alpha_{2}$-macroglobulin. Any observed hydrolysis of BAPNA was thus due to $\alpha_{2}$-macroglobulin-bound trypsin. In control experiments, the hydrolytic activity of trypsin-free, papain- $\alpha_{2}$-macroglobulin complex in the presence of iodoacetate was found to be negligible. The rate of hydrolysis of BAPNA by the papain + trypsin: $\alpha_{2}$-macroglobulin reaction mixtures was thus a measure of $\alpha_{2}$-macroglobulin-bound trypsin. The data indicated that only about $10 \%$ of the $\alpha_{2}$-macroglobulin-bound proteinase was trypsin. When the trypsin:papain molar ratio was increased to 10:1 approximately $50 \%$ of the bound proteinase was trypsin.

In parallel studies in which Azocoll was used to monitor the unbound papain concentration, the $\alpha_{2}$-macroglobulin was also found to bind papain approximately 10 times faster than trypsin. In these studies any unbound trypsin was inactivated by the addition of soybean trypsin inhibitor; no iodoacetate was added to the reaction mixtures.

\section{DISCUSSION}

The recent studies of Barrett et al. (26, 27), of Sottrup-Jensen et al. (28-33), and of others have suggested that the interaction of $\alpha_{2}$-macroglobulin with proteinases involves several events. The interaction is initiated by binding of the proteinase at its active site to the "bait" region of the $\alpha_{2}$-macroglobulin peptide chain. This interaction results in the cleavage of a proteinase-labile peptide bond near the middle of the $\alpha_{2}$-macroglobulin peptide chain $(29,32)$. Peptide bond cleavage is followed by a change in the conformation of the $\alpha_{2}$ macroglobulin which becomes more compact. During or after this conformational change, the proteinase binds to a different site on the $\alpha_{2}$-macroglobulin. This interaction does not involve the active site of the proteinase which remains catalytically active. This terminal interaction may involve the unique glutamyl-cysteinyl thioester linkage in the $\alpha_{2}$-macroglobulin which apparently becomes much more reactive after either cleavage of the $\alpha_{2}$-macroglobulin peptide chain or the conformational change has occurred.

This pathway has been based largely on data obtained on the interaction of trypsin with $\alpha_{2}$-macroglobulin. Per se, it does not explain the differences which have been observed in the binding ratios of the various protcinase- $\alpha_{2}$-macroglobulin complexes.

All available data show that at least one molecule of a reactive proteinase is bound in a stable complex by $\alpha_{2}$-macroglobulin. The variability only arises in the extent to which a second proteinase molecule can be bound.

The binding and SDS-gel electrophoresis studies reported in this paper strongly suggest that there is a parallelism between the stoichiometry of the saturated complex and the extent to which the proteinase is able to cleave the scissile $\alpha_{2}$ macroglobulin peptide bonds. Trypsin (stoichiometry $=2: 1$ ) cleaves all four of the scissile $\alpha_{z}$-macroglobulin peptide bonds; chymotrypsin ( $\mathrm{pH} 7.65$, stoichiometry $=1.4: 1$ ) cleaves about two-thirds of the scissile bonds; plasmin (stoichiometry $=1: 1$ ) cleaves about half of the scissile bonds. In other words, if the second proteinase molecule cannot bind to and cleave the "bait" region of the two intact $\alpha_{2}$-macroglobulin chains still present in the 1:1 proteinase- $\alpha_{2}$-macroglobulin complex it is unable to bind to $\alpha_{2}$-macroglobulin at the sites which characterize the stable, endproduct complex.

The studies on the rate of reaction of the four proteinases with $\alpha_{2}$-macroglobulin suggest that the stoichiometry of the saturated proteinase- $\alpha_{2}$-macroglobulin complex is directly related to the rate of formation of the transient active site complex. Trypsin (stoichiometry $=2: 1$ ) is bound by $\alpha_{2}$-macroglobulin 10 times slower than papain (stoichiometry $=2: 1$ ), 4 times faster than chymotrypsin (pH 7.65, stoichiometry $=1.4: 1$ ), and 100 times faster than plasmin (stoichiometry $=1: 1$ ).

If $\alpha_{2}$-macroglobulin undergoes a major 
conformational change upon interaction with a proteinase as proposed by Barrett (3), and if this "fast" form $\alpha_{2}$-macroglobulin can no longer bind proteinase, as has been noted for trypsin by Van Leuven (34), it is reasonable to propose that the stoichiometry of binding for a particular proteinase will be related to its rate of binding to $\alpha_{2}$-macroglobulin relative to the rate of the $\alpha_{2}$-macroglobulin conformational change. Thus, if the rate of the initial proteinasc: $\alpha_{\mathrm{z}}$-macroglobulin interaction is fast relative to that of the conformational change, the "native" conformation of $\alpha_{2}$ macroglobulin will bind two molecules of proteinase. If the rate of the proteinase: $\alpha_{2}$ macroglobulin interaction approaches that of the conformational change then only partial occupancy of the second binding site will occur since a proportion of the $\alpha_{2^{-}}$ macroglobulin molecules will have undergone the conformational change before the second proteinase molecule can bind to the $\alpha_{2}$-macroglobulin. If the rate of binding of proteinase is slow relative to that of the conformational change, only one proteinase molecule will be bound. For example, the rate of formation of the plasmin- $\alpha_{2^{-}}$ macroglobulin complex is relatively slow and a stoichiometry of $1: 1$ is obtained for this complex.

This hypothesis can explain (i) the lower proteinase binding ratios that are associated with the slower chymotrypsin and plasmin reactions (relative to trypsin), (ii) the lower stoichiometry of partially saturated trypsin- and chymotrypsin- $\alpha_{2^{-}}$ macroglobulin complexes to which excess trypsin or chymotrypsin had been added later, and (iii) the effect of $\mathrm{pH}$ on the stoichiometry of the chymotrypsin- $\alpha_{2}$-macroglobulin complex but not of the trypsin complex. If the rate of the trypsin interaction is sufficiently faster than that of the conformational change, the relatively small changes in the rate of binding which are induced by varying the $\mathrm{pH}$ from 9 to 5 should not measurably affect the stoichiometry of the complex. If however the rate of binding of chymotrypsin to $\alpha_{2}$-macroglobulin is sufficiently close to that of the conformational change, changes in $\mathrm{pH}$ could sufficiently alter the relative rates of these two reactions such that the stoichiometry of the stable, end-product complex would be $\mathrm{pH}$ dependent.

\section{REFERENCES}

1. Hald, P. R., AND Roberts, R. C. (1978) Biochem. J. 173, 27-38.

2. HARPEL, P. C. (1973) J. Exp. Med. 138, 508-521.

3. BAKKETT, A. J., AND S'lAKKEY, P. M. (1973) Biochem. J. 133, 709-724.

4. HARPel, P. C., AND Rosenberg, R. D. (1976) in Progress in Hemostasis and Thrombosis (Spaet, R. H., ed.), Vol. 3, $\alpha_{2}$ Macroglobulin and Antithrombin-Heparin Cofacter: Modulators of Hemostatic and Inflammatory Reactions, pp. 145-189, Grune \& Stratton, New York.

5. Harpel, P. C., AND Mosesson, M. W. (1973) $J$. Clin. Invest. 52, 2175-2184.

6. Ganrot, P. O. (1967) Acta Chem. Scand 21, 602608.

7. SAunders, R., Dyce, B. J., V ANNier, W. E., AND HAVERBACK, B. J. (1971) J. Clin. Invest. 50, 23762383.

8. Schreiber, A. D., Kaplan, A. P., and Austen, K. F. (1973) J. Clin. Invest. 42, 1394-1401.

9. Pochon, F., Amand, B., ANd LavaletTe, D. (1978) J. Biol Chem. 253, 7496-7499.

10. Deutsch, D. G., AND MeRTZ, E. T. (1970) Science 170, 1095-1096.

11. WIMAN, B., AND W ALLEN, P. (1973) Eur. J. Biochem. 36, 25-31.

12. Burke, D. E., Lewis, S. D., AND Shafer, J. A. (1974) Arch. Biochem. Biophys. 164, 30-36.

13. KLEIN, M., AND MiHAEsCo, C. (1973) Biochem. Biophys. Res. Commun. 52, 774-778.

14. HALL, P. R., AND RoBERTS, R. C. (1978) Biochem. J. 173, 27-38.

15. Chase, T., AND Shaw, E. (1967) Biochem. Biophys. Res. Commun 29, 508-514.

16. Chase, T., AND Shaw, E. (1969) Biochemistry 8, 2212-2224.

17. Bender, M. L., Begue-Canton, M. L., Blakeley, R. L., Brubacher, L. J., Feder, J., Gunter, C. R., Kezoy, F. J., Killheffer, J. V., JR., MARShald, T. H., Milleer, C. G., Roeske, R. W., AND Stoops, J. L. (1966) J. Amer. Chem. Soc. 88, 5890-5913.

18. Nicholson, E. M., AND Shafer, J. A. (1980) Arch. Biochem. Biophys. 200, 560-566.

19. BROCKLehURST, K., AND LITTLE, G. (1973) Biochem. J. 133, 67-80.

20. SAUNDERS, R. (1970) Ph.D. Dissertation, University of Southern California.

21. Bloom, J. W., ANd Hunter, M. J. (1978) J. Biol Chem. 253, 547-559.

22. WeBer, K., AND Osborn, M. (1969) J. Biol. Chem. 244, 4406-4412. 
23. Harpel, P. C., Hayes, M. B., and Hugli, T. E. (1979) J. Biol Chem. 254, 8669-8678.

24. Pochon, F., Amand, B., Lavalette, D., AND Bieth, J. (1978) J. Biol Chem. 253, 7496-7499.

25. Wang, O., WU, K., and FeINMan, R. D. (1981) Arch. Biochem. Biophys. 211, 500-506.

26. Barrett, A. J., Brown, M. A., AND SAyers, C. A. (1979) Biochem. J. 181, 401-418.

27. Salvesen, G. S., Sayers, C. A., and Barrett, A. J. (1981) Biochem. J. 195, 453-461.

28. Sottrup-Jensen, L., Petersen, T. E., and MagNUSSON, S. (1980) FEBS Lett. 121, 275-279.

29. SotTRUP-JENSEN, L., HANSEN, H. F., MoRTENSEN,
S. B., Petersen, T. E., and Magnusson, S. (1981) FEBS Lett. 123, 145-148.

30. SotTRUP-JENSEN, L., Longlad, P. B., StEPhanik, T. M., Petersen, T. E., Magnusson, S., AND JoRNVALL, H. (1981) FEBS Lett. 127, 167-173.

31. Sottrup-Jensen, L., Petersen, T. E., AND MaGNUSSON, S. (1981) FEBS Lett. 128, 123-126.

32. Sottrup-Jensen, L., Petersen, T. E., AND MaGNUSSON, S. (1981) FEBS Lett. 128, 127-132.

33. Mortensen, S. B., SotTrip-Jfinsen, L., Hansen, H. F., Petersen, T. E., and Magnusson, S. (1981) FEBS Lett. 135, 295-300.

34. Van Leuven, F., Cassiman, J. J., and Van Den BERGHE, H. (1982) Biochem. J. 201, 119-128. 